\section{Pulmonary arteriovenous malformations emerge from the shadows}

\author{
Claire L Shovlin, ${ }^{1,2}$ Robin Condliffe, ${ }^{3,4}$ James W Donaldson, ${ }^{5}$ \\ David G Kiely, ${ }^{3,4}$ Stephen J Wort ${ }^{1,6}$
}

For many years, pulmonary arteriovenous malformations (PAVMs) have been an underdiagnosed and poorly understood aspect of respiratory medicine. Recent increases in the published literature have improved our understanding of their diagnosis and management. The British Thoracic Society Clinical Statement on PAVMs published in Thorax aims to drive an improvement in the care provided to this often-neglected patient group. ${ }^{1}$ The Statement is the first example of a new method of summarising data and providing an expert view of best practice in a given clinical area.

Three major issues are associated with poor health outcomes in patients with PAVMs: lack of clinician awareness and education, an absence of standardised approaches to patient management and an urgent need for service development for a condition estimated to affect more than 10000 people in the UK. ${ }^{2}$

\section{CLINICIAN AWARENESS AND EDUCATION}

PAVMs are under-represented in medical school syllabuses and, more importantly, not mentioned in UK or US specialist respiratory training curriculae. ${ }^{3}$ These abnormal blood vessels connect pulmonary arteries directly to pulmonary veins, allowing a proportion of systemic venous blood to bypass the pulmonary capillary bed and return 'unprocessed' to the left heart and systemic arterial tree (figure 1). Although shunting via PAVMs

\footnotetext{
${ }^{1} \mathrm{NHLI}$ Vascular Science, Imperial College London, London, UK

${ }^{2}$ Respiratory Medicine, and VASCERN HHT European Reference Centre, Hammersmith Hospital, Imperial College Healthcare NHS Trust, London, UK ${ }^{3}$ Pulmonary Vascular Disease Unit, Royal Hallamshire Hospital, Sheffield, UK

${ }^{4}$ Department of Infection, Immunity and Cardiovascular Disease, University of Sheffield, Sheffield, UK

${ }^{5}$ Respiratory Medicine, Derby Teaching Hospitals NHS Foundation Trust, Derby, UK

${ }^{6}$ Pulmonary Hypertension Department, Royal Brompton Hospital, London, UK
}

Correspondence to Professor Claire L Shovlin, Professor of Practice (Clinical and Molecular Medicine), NHLI Vascular Science, Imperial College London, Imperial Centre for Translational and Experimental Medicine, Hammersmith Campus, Du Cane Road, LondonW12 ONN, UK; c.shovlin@imperial.ac.uk impairs gas exchange, patients usually compensate very well for hypoxaemia and the exuberant ventilatory requirements by physiological mechanisms such as secondary erythrocytosis and changes in haemodynamics to maintain normal oxygen delivery (figure 2). In contrast, there are no compensatory mechanisms that enable restoration of the pulmonary capillary 'filter' to prevent paradoxical emboli (figure 2). ${ }^{4}$

In the UK, PAVM complication rates remain particularly high $^{5}: 6 \%$ cerebral abscess, ${ }^{6} 12 \%$ ischaemic stroke ${ }^{7}$ and $1 \%$ maternal death in pregnancy. ${ }^{8}$ It is of concern that this may be partly driven by poor appreciation of the condition-one recent UK study found that the mean delay from diagnosis to referral for treatment was 7.5 years. ${ }^{9}$ Conversely, a lack of experience with the condition can lead to patients receiving inaccurate or misleading counsel regarding complication risks (eg, overestimating bleeding risk), which can cause unnecessary alarm.

Hereditary haemorrhagic telangiectasia (HHT) is found in most patients with PAVMs, and may be diagnosed clinically using the Curaçao Criteria. ${ }^{10}$ Only if the PAVM is single should a sporadic aetiology be considered; even then, a single PAVM is most likely to be due to HHT. HHT is a multisystemic vascular disorder, inherited as an autosomal dominant trait and usually caused by a pathogenic gene variant ('mutation') in ENG, ACVRL1 or SMAD4. ${ }^{11}$ Almost all individuals with HHT experience recurrent nosebleeds due to nasal telangiectasia, and these frequently lead to iron deficiency and anaemia, sometimes enhanced by blood loss from gastrointestinal telangiectasia. ${ }^{12}$ Approximately $50 \%$ of patients with HHT have PAVMs, similar proportions have hepatic AVMs and $10 \%$ are estimated to have cerebral AVMs. A high clinical index of suspicion for HHT/PAVMs is crucial to case ascertainment-despite this, HHT passes unmentioned in many formal clinician training programmes. At least one European country has initiated programmes that have normalised life expectancy in HHT. ${ }^{13}$ However, across the UK, barely $10 \%$ of the expected number of HHT cases are seen in specialist centres, and life expectancy is estimated to be reduced by 2 years implying that complication rates are likely to be higher. ${ }^{14}$

\section{STANDARDISED APPROACH TO CLINICAL MANAGEMENT}

This British Thoracic Society Clinical Statement ${ }^{1}$ addresses PAVM management in adults, from basic principles through state-of-the-art, evidence-based updates to earlier clinical guidance. Key points include:

- It is critical that individuals with PAVMs are referred to services capable of providing comprehensive counselling and care, given the evidence that the major complications of PAVMs can be prevented and general lifestyle and supportive therapy can improve patient outcomes.

- Embolisation remains the mainstay of interventional treatment, but the prevention of major complications is also aided by general measures that need to be followed throughout the life of the patient. An important example is the use of antibiotic prophylaxis prior to dental procedures. ${ }^{15}$

- The risk-benefit profile and the cost of various follow-up approaches must also be carefully considered. For

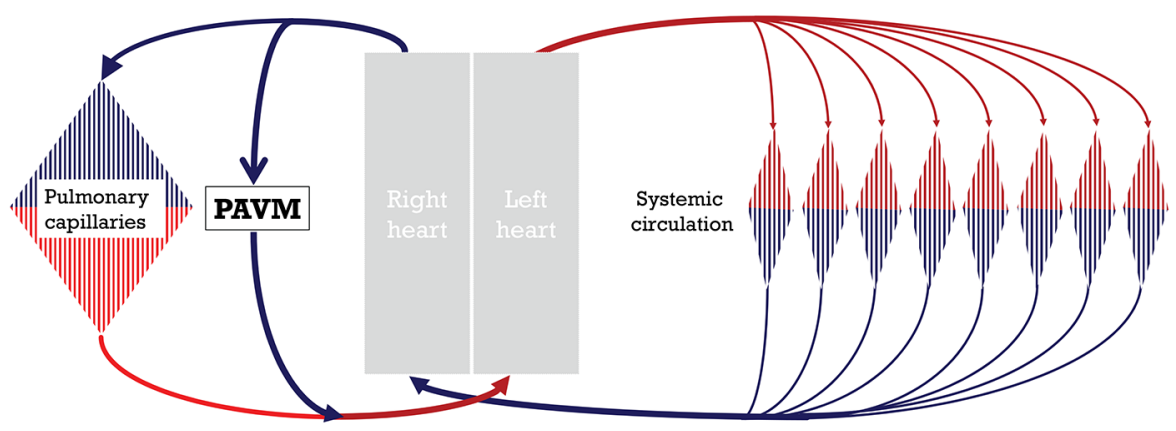

Figure 1 The impact of PAVMs on the circulation. A pulmonary arteriovenous malformation (PAVM) provides an anatomic right-to-left shunt, allowing unprocessed pulmonary arterial blood to enter the systemic circulation. 


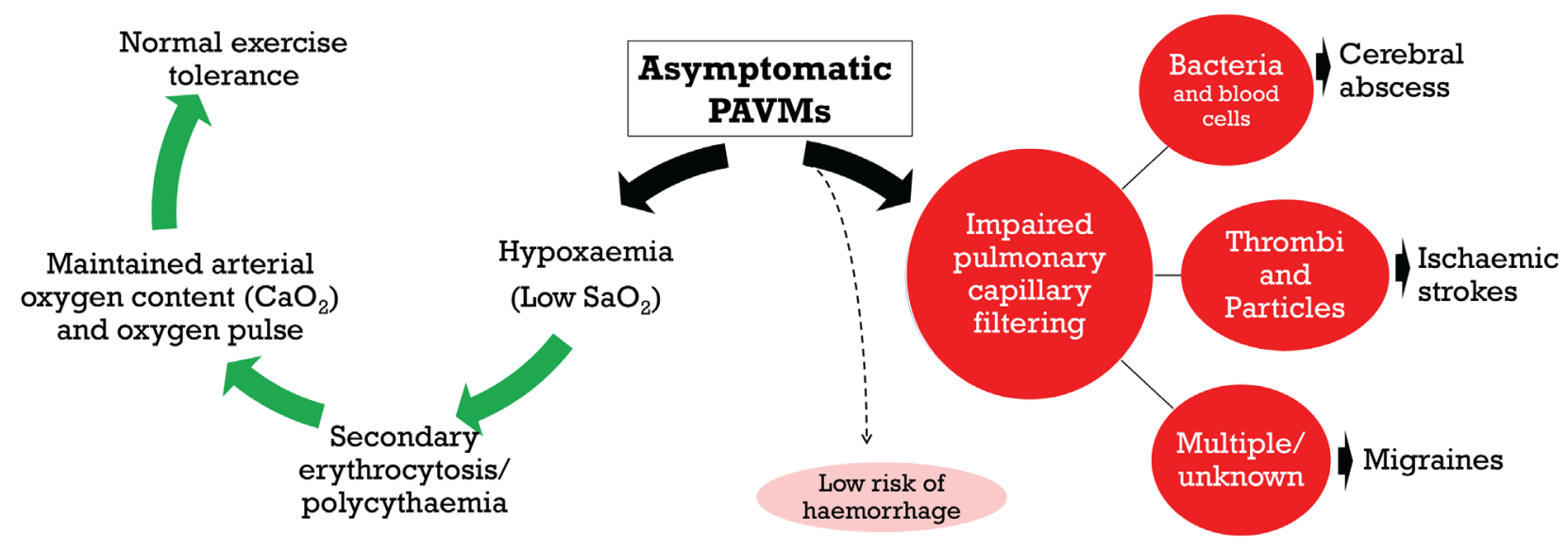

Figure 2 Pulmonary arteriovenous malformation (PAVM) pathophysiology. Slower compensatory processes on the left side of the diagram contrast with 'critical events' of decompensation on the right.

example, there has been a move away from protocolised CT scan follow-up in view of cumulative radiation dosages. ${ }^{16}$

- Patients with HHT should be screened for PAVMs in adult life. Adult children, siblings and parents of patients with PAVM(s) and/or HHT should be screened for HHT and PAVMs. When performing PAVM screening, a normal chest X-ray and oxygen saturations are not sufficient to exclude PAVMs, and either a normal thoracic CT scan or negative contrast echocardiogram is required, the exact choice of which will depend on patient's circumstances as detailed in the full statement.

\section{SERVICE DEVELOPMENT}

The third, and arguably most challenging area to address, is how to develop an appropriately supported service throughout the UK to be able to meet one of the aims of the NHS in giving equitable and timely access to high-quality care for all patients. For example, the largest UK PAVM service has managed more than 800 patients with PAVMs and more than 400 HHT families since its inception in 1984. Referrals now exceed 300 cases per annum, but the consultant-led service receives no specific commissioning support. Services in smaller PAVM/HHT centres in other parts of the country are also delivered by clinicians without specific commissioning support.

Within the NHS, there is a lack of recognition of the long term and complex nature of care that these patients require. PAVMs and HHT may be perceived as too common for specialist service commissioning, but service hubs are too infrequent for commissioning to be addressed regionally. Delivery of good clinical care requires robust links between locally based and specialist care. In turn, in light of the multisystem nature of HHT, specialist centres need to be embedded within a clinical network encompassing multiple medical and surgical specialities.

Encouragingly, there are indicators that PAVMs are finally receiving the necessary attention that may lead to such systems being realised. The European Commission has led the way in establishing the European Reference Network for Rare Multisystemic Vascular Diseases (VASCERN). ${ }^{17}$ VASCERN is a natural home for PAVMs and HHT, although currently includes only one UK PAVM/HHT service. ${ }^{18}$ The patient pathways recommended by VASCERN are based on intermittent review of patients in specialist centres, with those centres supporting local and primary care providers of long-term care. In addition, NHS England's new gene test commissioning pathways for mainstream clinicians should help guide familial management in conditions such as PAVMs and HHT which may be difficult to diagnose clinically. Finally, it is hoped that the championing of the condition by the British Thoracic Society and the provision of the Clinical Statement will influence the NHS to adequately finance the service that patients with PAVMs and HHT deserve.

In summary, we hope that the accompanying Clinical Statement ${ }^{1}$ will shine a spotlight on this underappreciated condition and bring PAVMs out of the shadows.

Contributors CLS was the lead author and responsible for the content overall, and Figures. RC, JWD, DGK and SJW reviewed, modified and approved the document. All authors agreed for the final submission.
Competing interests None declared.

Provenance and peer review Commissioned; externally peer reviewed.

(c) Article author(s) (or their employer(s) unless otherwise stated in the text of the article) 2017. All rights reserved. No commercial use is permitted unless otherwise expressly granted.

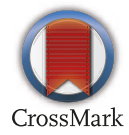

To cite Shovlin CL, Condliffe R, Donaldson JW, et al. Thorax 2017;72:1071-1073.

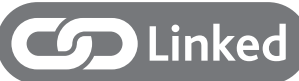

- http://dx.doi.org/10.1136/thoraxjnl-2017-210764

Thorax 2017;72:1071-1073.

doi:10.1136/thoraxjnl-2017-211072

\section{REFERENCES}

1 Shovlin CL, Condliffe R, Donaldson JW, et al. British Thoracic Society Clinical Statement on Pulmonary Arteriovenous Malformations. Thorax 2017:72:1154-63.

2 Nakayama M, Nawa T, Chonan T, et al. Prevalence of pulmonary arteriovenous malformations as estimated by low-dose thoracic CT screening. Intern Med 2012:51:1677-81.

3 Shovlin CL, Gossage JR. Pulmonary arteriovenous malformations: evidence of physician under-education. ERJ Open Res 2017;3:00104-2016.

4 Butler BD, Hills BA. The lung as a filter for microbubbles. J Appl Physiol 1979;47:537-43.

5 Donaldson JW, McKeever TM, Hall IP, et al. Complications and mortality in hereditary hemorrhagic telangiectasia: a population-based study. Neurology 2015;84:1886-93.

6 Boother EJ, Brownlow S, Tighe HC, et al. Cerebral abscess associated with odontogenic bacteremias, hypoxemia, and iron loading in immunocompetent patients with right-to-left shunting through pulmonary arteriovenous malformations. Clin Infect Dis 2017;65:595-603. 
7 Shovlin CL, Chamali B, Santhirapala V, et al. Ischaemic strokes in patients with pulmonary arteriovenous malformations and hereditary hemorrhagic telangiectasia: associations with iron deficiency and platelets. PLoS One 2014;9:e88812.

8 Shovlin CL, Sodhi V, McCarthy A, et al. Estimates of maternal risks of pregnancy for women with hereditary haemorrhagic telangiectasia (Osler-Weber-Rendu syndrome): suggested approach for obstetric services. BJOG 2008:115:1108-15.

9 Shovlin CL, Jackson JE, Bamford KB, et al. Primary determinants of ischaemic stroke/brain abscess risks are independent of severity of pulmonary arteriovenous malformations in hereditary haemorrhagic telangiectasia. Thorax 2008:63:259-66.

10 Shovlin CL, Guttmacher AE, Buscarini E, et al. Diagnostic criteria for hereditary hemorrhagic telangiectasia (Rendu-Osler-Weber syndrome). Am J Med Genet 2000;91:66-7.
11 McDonald J, Wooderchak-Donahue W, VanSant Webb C, et al. Hereditary hemorrhagic telangiectasia: genetics and molecular diagnostics in a new era. Front Genet 2015:6:1.

12 Finnamore H, Le Couteur J, Hickson M, et al. Hemorrhage-adjusted iron requirements, hematinics and hepcidin define hereditary hemorrhagic telangiectasia as a model of hemorrhagic iron deficiency. PLoS One 2013;8:e76516.

13 Kjeldsen A, Aagaard KS, Tørring PM, et al. 20-year follow-up study of Danish HHT patients-survival and causes of death. Orphanet $J$ Rare Dis 2016:11:157.

14 Donaldson JW, McKeever TM, Hall IP, et al. The UK prevalence of hereditary haemorrhagic telangiectasia and its association with sex, socioeconomic status and region of residence: a population-based study. Thorax 2014;69:161-7.
15 Limeres Posse J, Álvarez Fernández M, Fernández Feijoo J, et al. Intravenous amoxicillin/clavulanate for the prevention of bacteraemia following dental procedures: a randomized clinical trial. J Antimicrob Chemother 2016;71:2022-30.

16 Hanneman K, Faughnan ME, Prabhudesai V. Cumulative radiation dose in patients with hereditary hemorrhagic telangiectasia and pulmonary arteriovenous malformations. Can Assoc Radiol J 2014:65:135-40.

17 European Reference Network for Rare Multisystemic Vascular Diseases (VASCERN). 2017 https://vascern. eu/ (accessed 19 Sep 2017).

18 European Reference Network for hereditary haemorrhagic telangiectasia (VASCERN HHT). 2017 https://vascern.eu/expertise/rare-diseases-wgs/hht-wg/ (accessed 19 Sep 2017). 